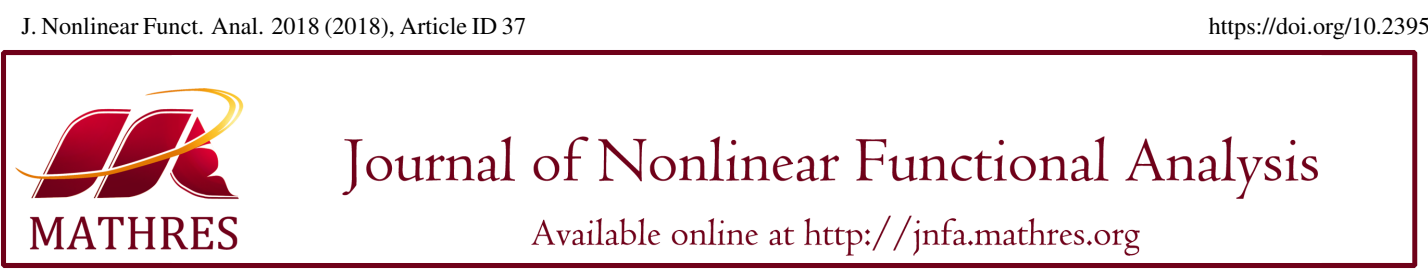

https://doi.org/10.23952/jnfa.2018.37

\title{
POSITIVE SOLUTIONS FOR SOME NONLOCAL ELASTIC BEAM EQUATION BOUNDARY VALUE PROBLEMS WITH FULLY NONLINEAR TERM
}

\author{
XIAOJUAN LIU ${ }^{1}$, ZHONGMIN SUN $^{2}$, YONGFANG WEI ${ }^{1}$, ZHANBING BAI $^{1, *}$ \\ ${ }^{1}$ College of Mathematics and System Science, Shandong University of Science and Technology, Qingdao 266590, China \\ ${ }^{2}$ School of Electromechanical Engineering, Weifang Engineering Vocational College, Qingzhou 262500, China
}

\begin{abstract}
This paper is concerned with the existence of at least one positive solution for the nonlocal elastic beam equation boundary value problem with fully nonlinear term. Our result is based on a fixed point theorem in a cone and the Green's function of the nonlocal elastic beam equation boundary value problem. An example is also presented to illustrate our results. Keywords. Elastic beam equation; Boundary value problem; Fixed-point theorem; Positive solution.
\end{abstract}

2010 Mathematics Subject Classification. 34B15.

\section{INTRODUCTION}

In this paper, we discuss the following nonlocal elastic beam equation boundary value problem with fully nonlinear term

$$
\begin{gathered}
y^{(4)}(x)+\delta y^{\prime \prime}(x)=f\left(x, y(x), y^{\prime}(x), y^{\prime \prime}(x), y^{\prime \prime \prime}(x)\right), 0<x<1, \\
y(0)=y(1)=\int_{0}^{1} p(x) y(x) d x, y^{\prime \prime}(0)=y^{\prime \prime}(1)=\int_{0}^{1} q(x) y^{\prime \prime}(x) d x,
\end{gathered}
$$

where we assume that

$\left(H_{1}\right) 0<\delta<\pi^{2}$

$\left(H_{2}\right) f:[0,1] \times R^{4} \rightarrow R^{+}$is continuous, $p, q \in L[0,1], p(x) \geq 0, q(x) \geq 0$, and

$$
\int_{0}^{1} q(x) \sin \sqrt{\delta} x d x+\int_{0}^{1} q(x) \sin \sqrt{\delta}(1-x) d x<\sin \sqrt{\delta}
$$

Recently, some results on the existence of solutions of the ordinary differential equation boundary value problem were established via different methods; see [1]-[15] and the references therein. In mechanics, the fourth-order boundary value problem is called the elastic beam equation which describes the deformation of an elastic beam in equilibrium state. In real problems, only positive solutions are meaningful.

${ }^{*}$ Corresponding author.

E-mail addresses: 17853273926@163.com (X. Liu), szm6970@sina.com (Z. Sun), weiyonfang@163.com (Y. Wei), zhanbingbai@163.com (Z. Bai).

Received July 10, 2018; Accepted October 17, 2018.

(C)2018 Journal of Nonlinear Functional Analysis 
The existence of positive solutions for the fourth-order differential equations with one or two parameters has received much attention in recent years. Bai [1] proved the existence of positive solutions for the following fourth-order boundary value problem with two parameters

$$
y^{(4)}(x)+\beta y^{\prime \prime}(x)=\lambda f\left(x, y(x), y^{\prime \prime}(x)\right), 0<x<1,
$$

with boundary condition (1.2), where $\lambda>0,0<\beta<\pi^{2}, f \in C([0,1] \times[0, \infty) \times(-\infty, 0],[0, \infty))$, functions $p, q \in L[0,1], p(x) \geq 0$, and $q(x) \geq 0$ such that

$$
\int_{0}^{1} q(x) \sin \sqrt{\beta} x d x+\int_{0}^{1} q(x) \sin \sqrt{\beta}(1-x) d x<\sin \sqrt{\beta}
$$

By the Krasnosel'skii's fixed point theorem, the author proved that boundary value problems (1.3)-(1.2) has at least one positive solution.

In [2], Guo, Yang and Liang showed the existence of positive solutions to (1.1)-(1.2) by defining two positive continuous convex functionals. Based on a fixed point theorem due to Guo and Ge [3] and the nonlocal fourth-order BVPs Green function, some criteria for the existence of positive solutions were obtained; see [2] and the references therein.

In [4], Li studied the solvability of the fourth-order boundary value problem

$$
\begin{gathered}
y^{(4)}(x)=f\left(x, y(x), y^{\prime \prime}(x)\right), 0 \leq x \leq 1, \\
y(0)=y(1)=y^{\prime \prime}(0)=y^{\prime \prime}(1)=0,
\end{gathered}
$$

where $f:[0,1] \times R^{2} \rightarrow R$ is continuous. By the maximum principle, the author developed a monotone iterative method for the above boundary value problem (1.4)-(1.5), and obtained some existence and uniqueness results of problem (1.4)

In $[5,6]$, a novel efficient method for this kinds of fourth-order nonlinear boundary value problems was given, and the existence and uniqueness results of positive solution of problem (1.1)-(1.2) were obtained. These authors reduced the problem to two second-order problems and defined an operator $A$ for the right hand side function with solutions of the second-order problems. They reduced the problem to a operator equation. Under some easily verified conditions on this function in a specified bounded domain, they proved the contraction of the operator. This guarantees the existence and uniqueness of a solution of the problem and the convergence of an iterative method for finding it.

All the above results were established under the assumption that derivatives $y^{\prime}, y^{\prime \prime \prime}$ were not involved explicitly or Lipschitz condition was involved in the nonlinear term $f$. Inspired and motivated by the results mentioned above, we will study the existence of positive solutions for the nonlocal fourth-order boundary value problem (1.1)-(1.2).

The rest of the paper is arranged as follows. In Section 2, we list a lemma and a theorem, which play an important role in the proof of our main results. In Section 3, we study the existence of positive solutions for the nonlocal fourth-order boundary value problem (1.1)-(1.2). In Section 4, an example is given to illustrate our main results. 


\section{PRELIMINARIES}

Denote by $G_{1}(x, t), G_{2}(x, t)$ the Green's function of the following problems

$$
\begin{aligned}
& \left\{\begin{array}{l}
-y^{\prime \prime}(x)=0,0<x<1, \\
y(0)=y(1)=\int_{0}^{1} p(x) y(x) d x ;
\end{array}\right. \\
& \left\{\begin{array}{l}
-y^{\prime \prime}(x)-\delta y(x)=0,0<x<1, \\
y(0)=y(1)=\int_{0}^{1} q(x) y(x) d x .
\end{array}\right.
\end{aligned}
$$

Then

$$
\begin{aligned}
& G_{1}(x, t)=K_{1}(x, t)+m_{1} \int_{0}^{1} K_{1}(t, s) p(s) d s \\
& G_{2}(x, t)=K_{2}(x, t)+m_{2} \int_{0}^{1} K_{2}(t, s) q(s) d s
\end{aligned}
$$

where

$$
\begin{gathered}
K_{1}(t, s)= \begin{cases}t(1-s), & \text { for } 0 \leq t \leq s \leq 1, \\
s(1-t), & \text { for } 0 \leq s \leq t \leq 1,\end{cases} \\
K_{2}(t, s)= \begin{cases}\frac{\sin \sqrt{\delta} t \sin \sqrt{\delta}(1-s)}{\sqrt{\delta} \sin \sqrt{\delta}}, & \text { for } 0 \leq t \leq s \leq 1, \\
\frac{\sin \sqrt{\delta} \sin \sqrt{\delta}(1-t)}{\sqrt{\delta} \sin \sqrt{\delta}}, & \text { for } 0 \leq s \leq t \leq 1,\end{cases} \\
m_{1}=\frac{1}{1-\int_{0}^{1} p(x) d x}, \\
m_{2}=\frac{\sin \sqrt{\delta} x+\sin \sqrt{\delta}(1-x)}{\sin \sqrt{\delta}-\int_{0}^{1} q(x) \sin \sqrt{\delta} x d x-\int_{0}^{1} q(x) \sin \sqrt{\delta}(1-x) d x} .
\end{gathered}
$$

Lemma 2.1. ([1]) Suppose that $\left(H_{1}\right)$ and $\left(H_{2}\right)$ hold. Then, for each $g(x) \in C[0,1]$, the problem

$$
\left\{\begin{array}{l}
y^{(4)}(x)+\delta y^{\prime \prime}(x)=g(x), 0<x<1, \\
y(0)=y(1)=\int_{0}^{1} p(x) y(x) d x, y^{\prime \prime}(0)=y^{\prime \prime}(1)=\int_{0}^{1} q(x) y^{\prime \prime}(x) d x,
\end{array}\right.
$$

has a unique solution

$$
y(x)=\int_{0}^{1} \int_{0}^{1} G_{1}(x, t) G_{2}(t, \tau) g(\tau) d \tau d t
$$

Using (2.4), we get

$$
\begin{aligned}
& y^{\prime}(x)=\int_{x}^{1} \int_{0}^{1}(1-t) G_{2}(t, \tau) g(\tau) d \tau d t-\int_{0}^{x} \int_{0}^{1} t G_{2}(t, \tau) g(\tau) d \tau d t, \\
& y^{\prime \prime}(x)=-\int_{0}^{1} G_{2}(x, t) g(t) d t, \\
& y^{\prime \prime \prime}(x)=-\int_{0}^{1} \frac{\partial G_{2}(x, t)}{\partial x} g(t) d t .
\end{aligned}
$$

Our main results are based on a fixed point theorem due to Bai and Ge [7], which is a generalization of the fixed point theorem of cone expansion and compression of norm type. 
Suppose that $Y$ is a Banach space, $P \subset Y$ is a cone, and $\alpha_{i}: Y \rightarrow[0, \infty), i=1,2, \cdots, n$ are given continuous nonnegative functionals. There exist two positive constants $M_{1}$ and $M_{2}$ such that

$$
\begin{gathered}
\alpha_{i}(\lambda y) \leq \lambda \alpha_{i}(y), \text { for } y \in Y, \lambda \in[0,1], i=1,2, \cdots, n, \\
M_{1} \max _{i} \alpha_{i}(y) \leq\|y\| \leq M_{2} \max _{i} \alpha_{i}(y), \text { for } y \in Y .
\end{gathered}
$$

Let $L_{i}>r_{i}>0, i=1,2, \cdots, n$ be constants. Let

$$
\Omega_{1}=\left\{y \in Y \mid \alpha_{i}(y)<r_{i}, \text { for } i=1,2, \cdots, n\right\}
$$

and

$$
\Omega_{2}=\left\{y \in Y \mid \alpha_{i}(y)<L_{i}, \text { for } i=1,2, \cdots, n\right\}
$$

be two open subsets in $Y$. Moreover, for $i=1,2, \cdots, n$, we denote

$$
\begin{aligned}
C_{i} & =\left\{y \in Y \mid \alpha_{i}(y)=r_{i}, \alpha_{j}(y) \leq r_{j}, \text { for } j=1,2, \cdots, n ; j \neq i\right\}, \\
D_{i} & =\left\{y \in Y \mid \alpha_{i}(y)=L_{i}, \alpha_{j}(y) \leq L_{j}, \text { for } j=1,2, \cdots, n ; j \neq i\right\} .
\end{aligned}
$$

Theorem 2.2. ([8]) If $T: P \rightarrow P$ is a completely continuous operator satisfying:

$$
\begin{aligned}
& \left(S_{1}\right) \alpha_{i}(T y) \leq r_{i}, y \in C_{i} \cap P ; \alpha_{i}(T y) \geq L_{i}, y \in D_{i} \cap P ; \text { or } \\
& \left(S_{2}\right) \alpha_{i}(T y) \geq r_{i}, y \in C_{i} \cap P ; \alpha_{i}(T y) \leq L_{i}, y \in D_{i} \cap P ;
\end{aligned}
$$

for all $i=1,2, \cdots, n$, then $T$ has at least one fixed point in $\left(\overline{\Omega_{2}} \backslash \Omega_{1}\right) \cap P$.

\section{EXISTENCE OF POSITIVE SOLUTIONS}

Let $Y=C^{3}[0,1]$ be a Banach space equipped with the norm $\|u\|=\max _{i \in\{1,2,3,4\}}\left\{\max _{0 \leq x \leq 1}\left|y^{(i-1)}(x)\right|\right\}$. Set $P=\left\{y \in Y \mid y(x) \geq 0, y^{\prime \prime}(x) \leq 0, x \in[0,1]\right\}$ a cone. Define functionals

$$
\alpha_{i}(y)=\max _{0 \leq x \leq 1}\left|y^{(i-1)}(x)\right|, \text { for } y \in Y, i=1,2,3,4 .
$$

Then $\alpha_{i}: Y \rightarrow[0, \infty)$ are four continuous nonnegative functionals such that $\|y\|=\max _{i \in\{1,2,3,4\}}\left\{\alpha_{i}(y)\right\}$ and (2.5), (2.6) hold. The functions $G_{1}(x, t), G_{2}(x, t)$ are defined by (2.1), (2.2). By $\left(H_{1}\right)$ and $\left(H_{2}\right)$, it is clear that

$$
G_{1}(x, t) \geq 0, G_{2}(x, t) \geq 0, \text { for } 0 \leq x, t \leq 1 .
$$

Define an operator $T$ as follows:

$$
(T y)(x):=\int_{0}^{1} \int_{0}^{1} G_{1}(x, t) G_{2}(t, \tau) f\left(\tau, y(\tau), y^{\prime}(\tau), y^{\prime \prime}(\tau), y^{\prime \prime \prime}(\tau)\right) d \tau d t
$$

Obviously, any fixed point of (3.2) is a solution of (1.1)-(1.2). From (3.2), we get

$$
\begin{aligned}
(T y)^{\prime}(x) & =\int_{x}^{1} \int_{0}^{1}(1-t) G_{2}(t, \tau) f\left(\tau, y(\tau), y^{\prime}(\tau), y^{\prime \prime}(\tau), y^{\prime \prime \prime}(\tau)\right) d \tau d t \\
& -\int_{0}^{x} \int_{0}^{1} t G_{2}(t, \tau) f\left(\tau, y(\tau), y^{\prime}(\tau), y^{\prime \prime}(\tau), y^{\prime \prime \prime}(\tau)\right) d \tau d t \\
(T y)^{\prime \prime}(x) & =-\int_{0}^{1} G_{2}(x, t) f\left(t, y(t), y^{\prime}(t), y^{\prime \prime}(t), y^{\prime \prime \prime}(t)\right) d t \\
(T y)^{\prime \prime \prime}(x) & =-\int_{0}^{1} \frac{\partial G_{2}(x, t)}{\partial x} f\left(t, y(t), y^{\prime}(t), y^{\prime \prime}(t), y^{\prime \prime \prime}(t)\right) d t
\end{aligned}
$$


By property (3.1) and assumption $f>0$, for $y \in P$, we have

$$
(T y)(x) \geq 0,(T y)^{\prime \prime}(x) \leq 0, x \in[0,1] .
$$

These imply that $T: P \rightarrow P$ is completely continuous by Arzela-Ascoli's theorem.

Now, we prove our main existence theorem for problem (1.1)-(1.2).

For the sake of convenience, we denote

$$
\begin{aligned}
& M_{1}=\max _{x \in[0,1]} \int_{0}^{1} \int_{0}^{1} G_{1}(x, t) G_{2}(t, \tau) d \tau d t \\
& M_{2}=\max \left\{\int_{0}^{1} \int_{0}^{1}(1-t) G_{2}(t, \tau) d \tau d t, \int_{0}^{1} \int_{0}^{1} t G_{2}(t, \tau) d \tau d t\right\}, \\
& M_{3}=\max _{x \in[0,1]} \int_{0}^{1} G_{2}(x, t) d t, M_{4}=\max _{x \in[0,1]} \int_{0}^{1} \frac{\partial G_{2}(x, t)}{\partial x} d t .
\end{aligned}
$$

Theorem 3.1. Suppose that $\left(H_{1}\right),\left(H_{2}\right)$ hold, $f:[0,1] \times R^{4} \rightarrow R^{+}$is continuous and there exist eight constants $L_{i}>r_{i}>0, i=1,2,3,4$, such that

$$
\begin{aligned}
& \left(A_{1}\right) f\left(x, y_{1}, y_{2}, y_{3}, y_{4}\right) \geq \max _{i}\left\{\frac{r_{i}}{M_{i}}\right\}, \\
& \text { for }\left(x, y_{1}, y_{2}, y_{3}, y_{4}\right) \in[0,1] \times\left[0, r_{1}\right] \times\left[-r_{2}, r_{2}\right] \times\left[-r_{3}, 0\right] \times\left[-r_{4}, r_{4}\right] ; \\
& \left(A_{2}\right) f\left(x, y_{1}, y_{2}, y_{3}, y_{4}\right) \leq \min _{i}\left\{\frac{L_{i}}{M_{i}}\right\}, \\
& \text { for }\left(x, y_{1}, y_{2}, y_{3}, y_{4}\right) \in[0,1] \times\left[0, L_{1}\right] \times\left[-L_{2}, L_{2}\right] \times\left[-L_{3}, 0\right] \times\left[-L_{4}, L_{4}\right] .
\end{aligned}
$$

Then problem (1.1)-(1.2) has at least one positive solution y such that

$$
r_{i} \leq \max _{0 \leq x \leq 1}\left|y^{(i-1)}(x)\right|, \text { for some of } i \in\{1,2,3,4\}
$$

and

$$
\max _{0 \leq x \leq 1}\left|y^{(j-1)}(x)\right| \leq L_{j}, \text { for all of } j \in\{1,2,3,4\}
$$

Proof. First, we set two bounded open subsets in $Y$ as follows

$$
\begin{aligned}
& \Omega_{1}=\left\{y \in Y \mid \alpha_{i}(y)<r_{i}, i=1,2,3,4\right\}, \\
& \Omega_{2}=\left\{y \in Y \mid \alpha_{i}(x)<L_{i}, i=1,2,3,4\right\} .
\end{aligned}
$$

Moreover, for $i=1,2,3,4$, let

$$
\begin{aligned}
& C_{i}=\left\{y \in Y \mid \alpha_{i}(y)=r_{i}, \alpha_{j}(y) \leq r_{j}, j=1,2,3,4 ; j \neq i\right\}, \\
& D_{i}=\left\{y \in Y \mid \alpha_{i}(y)=L_{i}, \alpha_{j}(y) \leq L_{j}, j=1,2,3,4 ; j \neq i\right\} .
\end{aligned}
$$

Then, we prove $\alpha_{i}(T y) \geq r_{i}$ for $y \in C_{i} \cap P, i=1,2,3,4$. By $\left(A_{1}\right)$, for $y \in C_{1} \cap P$, we get

$$
\begin{aligned}
\alpha_{1}(T y) & =\max _{x \in[0,1]}|(T y)(x)| \\
& =\max _{x \in[0,1]} \int_{0}^{1} \int_{0}^{1} G_{1}(x, t) G_{2}(t, \tau) f\left(\tau, y(\tau), y^{\prime}(\tau), y^{\prime \prime}(\tau), y^{\prime \prime \prime}(\tau)\right) d \tau d t \\
& \geq \frac{r_{1}}{M_{1}} \cdot \max _{x \in[0,1]} \int_{0}^{1} \int_{0}^{1} G_{1}(x, t) G_{2}(t, \tau) d \tau d t \\
& =r_{1} .
\end{aligned}
$$


For $y \in P$, we have $(T y)^{\prime \prime}(x) \leq 0$, that is, $(T y)^{\prime}(x)$ is decreasing on $[0,1]$. Thus

$$
\max _{x \in[0,1]}\left|(T y)^{\prime}(x)\right|=\max \left\{\left|(T y)^{\prime}(0)\right|,\left|(T y)^{\prime}(1)\right|\right\} .
$$

For $y \in C_{2} \cap P$, by $\left(A_{1}\right)$ and $f \geq 0$, we have

$$
\begin{aligned}
\alpha_{2}(T y) & =\max _{x \in[0,1]}\left|(T y)^{\prime}(x)\right| \\
& =\max _{x \in[0,1]} \mid \int_{x}^{1} \int_{0}^{1}(1-t) G_{2}(t, \tau) f\left(\tau, y(\tau), y^{\prime}(\tau), y^{\prime \prime}(\tau), y^{\prime \prime \prime}(\tau)\right) d \tau d t \\
& -\int_{0}^{x} \int_{0}^{1} t G_{2}(t, \tau) f\left(\tau, y(\tau), y^{\prime}(\tau), y^{\prime \prime}(\tau), y^{\prime \prime \prime}(\tau)\right) d \tau d t \mid \\
& =\max \left\{\int_{0}^{1} \int_{0}^{1}(1-t) G_{2}(t, \tau) f\left(\tau, y(\tau), y^{\prime}(\tau), y^{\prime \prime}(\tau), y^{\prime \prime \prime}(\tau)\right) d \tau d t\right. \\
& \left.\int_{0}^{1} \int_{0}^{1} t G_{2}(t, \tau) f\left(\tau, y(\tau), y^{\prime}(\tau), y^{\prime \prime}(\tau), y^{\prime \prime \prime}(\tau)\right) d \tau d t\right\} \\
\geq & \frac{r_{2}}{M_{2}} \cdot \max \left\{\int_{0}^{1} \int_{0}^{1}(1-t) G_{2}(t, \tau) d \tau d t, \int_{0}^{1} \int_{0}^{1} t G_{2}(t, \tau) d \tau d t\right\} \\
& =r_{2} .
\end{aligned}
$$

For $y \in C_{3} \cap P$, it follows from $\left(A_{1}\right)$ that

$$
\begin{aligned}
\alpha_{3}(T y) & =\max _{x \in[0,1]}\left|(T y)^{\prime \prime}(x)\right| \\
& =\max _{x \in[0,1]}\left|-\int_{0}^{1} G_{2}(x, t) f\left(t, y(t), y^{\prime}(t), y^{\prime \prime}(t), y^{\prime \prime \prime}(t)\right) d t\right| \\
& \geq \frac{r_{3}}{M_{3}} \cdot \max _{x \in[0,1]} \int_{0}^{1} G_{2}(x, t) d t, \\
& =r_{3} .
\end{aligned}
$$

For $y \in P$, we have $(T y)^{(4)}(x) \geq 0$, that is, $(T y)^{\prime \prime \prime}(x)$ is increasing on $[0,1]$. Thus

$$
\max _{x \in[0,1]}\left|(T y)^{\prime \prime \prime}(x)\right|=\max \left\{\left|(T y)^{\prime \prime \prime}(0)\right|,\left|(T y)^{\prime \prime \prime}(1)\right|\right\} .
$$

Combining with $\left(A_{1}\right)$ and $f \geq 0$, for $y \in C_{4} \cap P$, we have

$$
\begin{aligned}
\alpha_{4}(T y) & =\max _{x \in[0,1]}\left|(T y)^{\prime \prime \prime}(x)\right| \\
& =\max _{x \in[0,1]}\left|-\int_{0}^{1} \frac{\partial G_{2}(x, t)}{\partial x} f\left(t, y(t), y^{\prime}(t), y^{\prime \prime}(t), y^{\prime \prime \prime}(t)\right) d t\right| \\
& \geq \frac{r_{4}}{M_{4}} \cdot \max _{x \in[0,1]} \int_{0}^{1} \frac{\partial G_{2}(x, t)}{\partial x} d t \\
& =r_{4} .
\end{aligned}
$$

Similarly, we can prove, for $y \in D_{i} \cap P, \alpha_{i}(T y) \leq L_{i}, i=1,2,3,4$. Using Theorem 2.2 yields that $T$ has a fixed point $y \in\left(\overline{\Omega_{2}}-\Omega_{1}\right) \cap P$ such that $y=T y$. It follows that problem (1.1)-(1.2) has at least one positive solution $u$ such that

$$
r_{i} \leq \max _{x \in[0,1]}\left|y^{(i-1)}(x)\right|, \text { and } \max _{x \in[0,1]}\left|y^{(j-1)}(x)\right| \leq L_{j}
$$


for some of $i=1,2,3,4$ and all $j=1,2,3,4$. The theorem is proved.

\section{An ExAmple}

Example 4.1. Consider the following boundary value problem

$$
\begin{gathered}
y^{(4)}(x)+\frac{\pi^{2}}{4} y^{\prime \prime}(x)=f\left(x, y(x), y^{\prime}(x), y^{\prime \prime}(x), y^{\prime \prime \prime}(x)\right), 0<x<1, \\
y(0)=y(1)=\int_{0}^{1} x y(x) d x, y^{\prime \prime}(0)=y^{\prime \prime}(1)=0,
\end{gathered}
$$

where

$$
f\left(x, y_{1}, y_{2}, y_{3}, y_{4}\right)=\frac{1}{50}\left(y_{1}+y_{3}\right)+\left|\cos \left(y_{2}+y_{4}\right)\right|+60 .
$$

In this problem, we know that $\delta=\pi^{2} / 4, p(x)=x, q(x)=0$. Then

$$
\begin{aligned}
& M_{1}=\frac{8(3 \sqrt{2}-4)}{\pi^{4}} \approx 0.02, M_{2}=\frac{8(\pi-2)}{\pi^{3}} \approx 0.29, \\
& M_{3}=\frac{4(\sqrt{2}-1)}{\pi^{2}} \approx 0.17, M_{4}=\frac{2}{\pi} \approx 0.64 .
\end{aligned}
$$

Taking $r_{1}=r_{2}=r_{3}=r_{4}=1, L_{1}=L_{2}=L_{3}=L_{4}=50$, we find that

$$
f\left(x, y_{1}, y_{2}, y_{3}, y_{4}\right) \geq 59 \geq \max _{i}\left\{\frac{r_{i}}{M_{i}}\right\}=\frac{r_{1}}{M_{1}} \approx 55,
$$

for $\left(x, y_{1}, y_{2}, y_{3}, y_{4}\right) \in[0,1] \times[0,1] \times[-1,1] \times[-1,0] \times[-1,1]$;

$$
f\left(x, y_{1}, y_{2}, y_{3}, y_{4}\right) \leq 57 \leq \min _{i}\left\{\frac{L_{i}}{M_{i}}\right\}=\frac{L_{4}}{M_{4}} \approx 78.125,
$$

for $\left(x, y_{1}, y_{2}, y_{3}, y_{4}\right) \in[0,1] \times[0,50] \times[-50,50] \times[-50,0] \times[-50,50]$. In other words, $f\left(x, y_{1}, y_{2}, y_{3}, y_{4}\right)$ satisfies $\left(A_{1}\right)$ and $\left(A_{2}\right)$ of Theorem 3.1, thus the boundary value problems (4.1) has at least one positive solution $y(x)$ satisfying

$$
r_{i} \leq \max _{0 \leq x \leq 1}\left|y^{(i-1)}(x)\right|, \max _{0 \leq x \leq 1}\left|y^{(j-1)}(x)\right| \leq L_{j},
$$

for some of $i=1,2,3,4$ and all of $j=1,2,3,4$.

\section{CONCLUSIONS}

In mechanics, the fourth-order boundary value problem is called a beam equation which describes the deformation of an elastic beam in equilibrium state. There are many results under the assumption that odd-order derivatives are not involved explicitly or Lipschitz condition can be involved in the nonlinear term. Our results are concerned with the existence of at least one positive solution for the nonlocal fourth-order boundary value problem with fully nonlinear term.

\section{Acknowledgments}

This paper was supported by NSFC (11571207), the Taishan Scholar project and and SDUST Graduate Innovation project SDKDYC170343. 


\section{REFERENCES}

[1] Z. Bai, Positive solutions of some nonlocal fourth-order boundary value problem, Appl. Math. Comput. 215 (2010), 4191-4197.

[2] Y. Guo, F. Yang, Y. Liang, Positive solutions for nonlocal fourth-order boundary value problems with all order derivatives, Bound. Value Probl. 2012 (2012), Article ID 29.

[3] Y. Guo, W. Ge, Positive solutions for three-point boundary value problems with dependence on the first order derivatives, J. Math. Anal. Appl. 290 (2004), 291-301.

[4] Y. Li, A monotone iterative technique for solving the bending elastic beam equations, Appl. Math. Comput. 217 (2010), 2200-2208.

[5] Q.A. Dang, Q.L Dang, T.K.Q. Ngo, A novel efficient method for nonlinear boundary value problems, Numer. Algorithms 76 (2017), 427-439.

[6] Q.A. Dang, T.K.Q. Ngo, Existence results and iterative method for solving the cantilever beam equation with fully nonlinear term, Nonlinear Anal. Real World Appl. 36 (2017) 56-68.

[7] Z. Bai, W. Ge, Existence of solutions to fourth-order quasilinear boundary value problem, Acta Math. Sinica 22 (2006), 1825-1830.

[8] A. Cabada, S. Tersian, Multiplicity of solutions of a two point boundary value problem for a fourth-order equation. Appl. Math. Comput. 219 (2013), 5261-5267.

[9] Y. Cui, J. Sun, Existence of multiple positive solutions for fourth-order boundary value problems in Banach spaces, Bound. Value Probl. 2012 (2012), Article ID 107.

[10] Y. Cui, Y. Zou, Existence and uniqueness theorems for fourth-order singular boundary value problems, Comput. Math. Appl. 58 (2009), 1449-1456.

[11] Y. Li, Existence of positive solutions for the cantilever beam equations with fully nonlinear terms, Nonlinear Anal. 27 (2016), 221-237.

[12] H. Ma, Symmetric positive solutions for nonlocal boundary value problems of fourth-order, Nonlinear Anal. 68 (2008), 645-651.

[13] Y. Pang, Z. Bai, Upper and lower solution method for a fourth-order four-point boundary value problem on time scales, Appl. Math. Comput. 215 (2009), 2243-2247.

[14] A. Domoshnitsky, Sturm theorems and distance between adjacent zeros for second order integro-differential equations, J. Nonlinear Var. Anal. 2 (2018), 155-164.

[15] Y. Zou, On the existence of positive solutions for a fourth-order boundary value problem, J. Funct. Spaces 2017 (2017), Article ID 4946198. 